\title{
Atividades educativas sobre a prática do aleitamento materno em uma unidade hospitalar do interior do Amazonas: um relato de experiência
}

\author{
Educational activities on the practice of breastfeeding in a hospital in the interior of Amazonas: an
} experience report

Actividades educativas sobre la práctica de la lactancia materna en un hospital del interior de Amazonas: un relato de experiencia

\author{
Sergio de Almeida Matos \\ ORCID: https://orcid.org/0000-0003-2066-9142 \\ Universidade Federal do Amazonas, Brasil \\ E-mail: sgalmeida0707@gmail.com \\ Raquel Peres de Oliveira \\ ORCID: https://orcid.org/0000-0001-5109-1940 \\ Universidade Federal do Amazonas, Brasil \\ E-mail: oliperes021@gmail.com \\ Ednei Pereira Parente \\ ORCID: https://orcid.org/0000-0001-9554-5115 \\ Universidade do Estado do Amazonas, Brasil \\ E-mail: eparente@uea.edu.br \\ Naiza Peres de Lima \\ ORCID: https://orcid.org/0000-0001-9657-177X \\ Universidade Federal do Amazonas, Brasil \\ E-mail: naiza_20@hotmail.com \\ Fernanda Katrine Pantoja Valério \\ ORCID: https://orcid.org/0000-0001-7431-6620 \\ Secretaria Municipal de Saúde de Coari, Brasil \\ E-mail: nandakatrine9@gmail.com \\ Josiane Montanho Mariño \\ ORCID: https://orcid.org/0000-0002-2876-0226 \\ Universidade Federal do Amazonas, Brasil \\ E-mail: enf_josiane@yahoo.com.br \\ Carlos Eduardo Bezerra Monteiro \\ ORCID: https://orcid.org/0000-0003-1787-1892 \\ Universidade Federal do Amazonas, Brasil \\ E-mail: edumonteiro_123@hotmail.com
}

\begin{abstract}
Resumo
O objetivo do trabalho é descrever a experiência vivenciada por discentes de enfermagem durante a prática de atividades educativas sobre o aleitamento materno em uma unidade hospitalar do interior do Amazonas. Trata-se de um estudo descritivo, do tipo relato de experiência, sobre a vivência de estudantes do $9^{\circ}$ período de enfermagem, ocorrido no período de 07 a 18 de outubro de 2019. O público beneficiado foi composto por puérperas do Alojamento Conjunto (AC) de parto normal e cesariana. Para a execução das atividades utilizou-se os cartazes das paredes dos ACs, sendo abordado a temática Aleitamento Materno. Para os acadêmicos esse momento resultou em um acréscimo de novas vivências, e aquisição de novas habilidades para a realização de educação em saúde. Enquanto as puérperas relataram que as ações em saúde voltadas para o aleitamento materno são fundamentais para adquirir conhecimento acerca da amamentação, leite materno e as orientações quanto ao uso de objetos que interfere na amamentação adequada da criança. Tal prática oportunizou um ganho único de aprimoramento profissional e pessoal, rico em informações e compartilhamento de experiências, que proporcionou conhecimento a mães-filhos e aos familiares gerando em um elo de confiança com esse público.
\end{abstract}

Palavras-chave: Enfermagem; Aleitamento materno; Alojamento conjunto; Educação em saúde.

\section{Abstract}

The objective of the work is to describe the experience lived by nursing students during the practice of educational activities on breastfeeding in a hospital in the interior of Amazonas. This is a descriptive study, of the experience report type, about the experience of students from the 9th nursing period, which took place from October 7 to 18 , 2019. The public benefited was composed of puerperal women of the Joint Accommodation (CA) of normal delivery 
and cesarean section. For the execution of the activities, posters on the walls of the ACs were used, with the theme of Breastfeeding being addressed. For academics, this moment resulted in an addition of new experiences, andacquisition of new skills for health education. While the puerperal women reported that health actions aimed at breastfeeding are essential to acquire knowledge about breastfeeding, breast milk and guidelines on the use of objects that interfere with the child's adequate breastfeeding. Such practice provided a unique gain of professional and personal improvement, rich in information and sharing of experiences, which provided knowledge to mother-children and family members, generating a bond of trust with this public.

Keywords: Nursing; Breast feeding; Rooming-in care; Health education.

\section{Resumen}

El objetivo del trabajo es describir la experiencia vivida por estudiantes de enfermería durante la práctica de actividades educativas sobre lactancia materna en un hospital del interior de Amazonas. Se trata de un estudio descriptivo, del tipo relato de experiencia, sobre la experiencia de estudiantes del $9^{\circ}$ período de enfermería, que se llevó a cabo del 7 al 18 de octubre de 2019. El público beneficiado estaba compuesto de puérperas de la Acomodación Conjunta (CA) de parto normal y cesárea. Para la ejecución de las actividades se utilizaron carteles en las paredes de las CC.AA., con el tema de Lactancia Materna. Para los académicos, este momento resultó en una adición de nuevas experiencias, yAdquisición de nuevas habilidades para la educación sanitaria. Mientras que las puérperas informaron que las acciones de salud dirigidas a la lactancia materna son fundamentales para adquirir conocimientos sobre lactancia materna, leche materna y pautas sobre el uso de objetos que interfieran con la adecuada lactancia materna del niño. Tal practica brindó una ganancia única de superación profesional y personal, rica en información e intercambio de experiencias, que brindó conocimiento a madres-hijos y familiares, generando un vínculo de confianza con este público.

Palabras clave: Enfermería; Lactancia materna; Alojamiento conjunto; Educación en salud.

\section{Introdução}

O Aleitamento Materno (AM) é um ato muito além de alimentar e nutrir a criança. É um método que vincula a integração de genitora/filho, que reflete no estado nutricional infantil, imunológico, fisiológico e no desenvolvimento cognitivo e emocional, bem como na saúde da criança ao longo da vida; além de contribuir positivamente na saúde física e emocional materna (Brasil, 2015).

O valor nutricional do leite materno, quando comparado a outras fontes, é comprovadamente superior. Estudos demonstram que o mesmo é capaz de garantir todos os nutrientes necessários para o desenvolvimento infantil adequado nos primeiros seis meses de vida. Além disso, os benefícios são inúmeros, tais como: redução de infecções respiratórias, alergias, diarreias, reduzindo o surgimento de Doenças Crônicas Não Transmissíveis (DCNTs), diminuindo os índices de mortalidade infantil e internações hospitalares (Brasil, 2015; Costa et al., 2013; Lima et al., 2018).

A Organização Mundial de Saúde (OMS) definiu os critérios de avaliação das práticas alimentares em crianças abaixo de dois anos, dito cujos indicadores do AM (OMS, 2008). Segundo Saldan et al. (2015), com as análises da cobertura de idade os indicadores foram atualizados em 2008 fazendo de algumas medidas opcionais e abrindo oportunidade de acrescentar outro indicador. Desta forma, os novos indicadores são: Aleitamento Materno Exclusivo (AME) abaixo de 6 meses, Aleitamento Materno Predominante (AMP) em menores de 6 meses, a continuidade do AM por um ano, continuidade por dois anos, duração dele, o indicador inserido do AM em idade apropriada e uso de mamadeira.

Considerando que um dos métodos de incentivo ao AM é o acolhimento através de medidas educativas, assistência ao pré-natal e puerpério imediato, a amamentação é percebida como um ato de amor, e não somente uma técnica, quando realizado de forma adequada. O processo da amamentação cria e fortalece o vínculo mãe-filho gerando assim, segurança psicoemocional e proporcionando saúde e bem-estar, em ambos (Souza et al., 2018).

As educações em saúde desenvolvidas inicialmente no pré-natal proporcionam conhecimento desde a gravidez, parto e puerpério, o que assegura o acompanhamento, a avaliação e a continuidade no atendimento. Nas ações educativas são abordados aspectos de bem-estar entre mãe/feto, incluindo a amamentação (Maeda et al., 2014).

No Brasil, o Alojamento Conjunto (AC) só veio acontecer em 1971 na capital Brasília-DF. Na metade dos anos 80, a 
OMS, o Ministério da Saúde e o Fundo de Emergência Internacional das Nações Unidas para a Infância (UNICEF) delegaram pela implementação do AC, tendo por fundamento o alto índice de desmame antes dos 6 meses no país (Pasqual et al., 2010). O AC é um sistema hospitalar que possibilita ao Recém-Nascido (RN) saudável a permanência de 24 horas por dia ao lado de sua genitora, até a alta hospitalar, permitindo a concretização de todos os cuidados assistenciais e auxiliando no estabelecimento de vínculo afetivo, além disso, este propicia um ambiente para o cumprimento de orientações à mãe e familiares sobre a saúde do binômio mãe-filho, objetivando prevenir infecções.

No ano de 2016, foram instituídas as diretrizes para as organizações da atenção integral e humanização para a mulher e ao RN no AC. Onde estabelece que a equipe multiprofissional deva promover e proteger o AM sob livre demanda, prestando ajuda as puérperas em momentos de dificuldades de acordo com suas necessidades; oferecer à puérpera informações sobre a importância de não dar nenhum outro alimento ou bebida, a não ser o leite materno, exceto em ocasiões que envolva prescrição médica ou nutricional que é disponibilizado através do banco de leite humano; também devem oferecer informações dos perigos da amamentação cruzada e do impedimento da mesma no AC (Brasil, 2016).

Nesse contexto, Coca et al. (2018) afirmam que há diversas sugestões para o estímulo ao AM, porém, muitas instituições particulares e públicas não seguem o programa devido a sua independência do hábito institucional e administração de seus índices de saúde. Todavia, percebe-se a sua utilização de modo aleatório, contrariando as existências de políticas de incentivo ao AM, como por exemplo: a Iniciativa Hospital Amigo da Criança (IHAC) que compreende a prática de incentivo ao AM é unanime para uma correta assistência à mãe e filho, objetivando prevenir o desmame precoce.

Segundo Lamounier et al. (2019) são muitas as instituições públicas e privada credenciadas no IHAC, mas nem todas conseguem cumprir os 10 passos proposto pela OMS e UNICEF, além de não cumprir as normas propostas pela Lei $n^{\circ} 11.265$ de 3 de janeiro de 2006 e revogada pela Lei Portaria $\mathrm{N}^{\circ} 1.153$ de 22 de maio de 2014, como por exemplo: as Normas Brasileira de Comercialização de Alimentos para Lactentes e Crianças de $1^{\text {a }}$ Infância, Bicos, Chupetas e Mamadeiras (NBCAL) como estratégia de promoção, proteção e apoio a saúde da criança e da mulher promovendo o sucesso no AM (Brasil, 2006; Brasil, 2014).

A atuação da equipe de enfermagem no processo de amamentação é de fundamental importância, visto que é possível identificar as dificuldades das mães em amamentar seus RNs, nesse sentido, o profissional enfermeiro atua como educador e facilitador no AM. As práticas de ações em saúde são essenciais para eliminar as incertezas e entraves que interferem na amamentação (Rocha et al., 2020).

Os conhecimentos técnicos e científicos da enfermagem são fundamentais na unidade do AC, seu conhecimento fortalece, aprimora, enriquece as estratégicas e o estimula a amamentação. $O$ enfermeiro atua unido a sua equipe multiprofissional da saúde realizando inúmeras práticas pertencentes ao zelo com mãe e filho, com ênfase as instruções diante das dificuldades psíquica, física e sociais através dos vínculos com o educador, executando intervenções apropriadas, visando a qualidade na assistência e no zelar (Silva et al., 2021)

Frente a isto, o objetivo do trabalho é descrever a experiência vivenciada por discentes de enfermagem durante a prática de atividades educativas sobre o aleitamento materno em um hospitalar do interior do Amazonas.

\section{Metodologia}

Trata-se de um estudo descritivo, do tipo relato de experiência, sobre a vivência obtida durante a disciplina Estágio Curricular Supervisionado (ECS) I, com estudantes do $9^{\circ}$ período do curso de enfermagem pertencente ao Instituto de Saúde e Biotecnologia (ISB) da Universidade Federal do Amazonas (UFAM).

O estágio ocorreu no período de 07 a 18 de outubro de 2019, no setor de AC em um hospital do interior do Amazonas, Brasil. A disciplina ECS I compõe a grade curricular do curso de enfermagem do ISB, perfazendo 420 horas ao todo. Desta 
carga horária, 105 horas foram cumpridas no setor de AC.

O público beneficiado por intermédio das atividades educativas foi composto por puérperas dos ACs de parto normal e cesariana. Para a execução das atividades utilizou-se os cartazes das paredes dos ACs, sendo abordado a temática AM. As puérperas encontravam-se acompanhadas de seus familiares, e essas atividades foram específicas para as mesmas.

Para o desenvolvimento do estágio curricular ficamos sob a preceptoria de um enfermeiro voluntário, vinculado ao referido hospital. As atividades foram desenvolvidas no setor de AC, composto por 01 posto de enfermagem, 04 salas, sendo 02 exclusivas para cesarianas e abortos, e outras 02 exclusivas para partos normais e emergências clínicas. A equipe do setor era composta por um médico pediatra, um médico ginecologista, uma enfermeira e técnicos de enfermagem.

No decorrer do estágio curricular foram realizadas diversas ações e procedimentos de enfermagem, como: gerenciamento de enfermagem, Sistematização da Assistência de Enfermagem (SAE), curativos, cateterismo vesical de demora, banho nos RNs. Além disso, foram desenvolvidas educações em saúde voltadas para mãe e filho, como: amamentação correta, amamentação cruzada, higiene corporal da criança, as vantagens do leite materno e cuidados com o coto umbilical. As atividades foram desenvolvidas junto à equipe de profissionais do setor de AC.

\section{Resultados e Discussão}

O ECS desenvolvido no AC foi uma oportunidade onde os discentes desenvolveram seus conhecimentos teóricos e habilidades técnicas atuando diretamente com as pacientes. Os alunos desempenharam suas atividades sob a ótica de um profissional de enfermagem exercendo suas competências assistenciais e gerencias. O ECS faz-se imprescindível para que os graduandos possam adquirir uma maior percepção da prática clínica obstétrica, agregando novos conhecimentos para a prática profissional e acadêmica.

Através das atividades educativas, resultou-se em um acréscimo de novas vivências para a realização de educação em saúde. A aproximação com os usuários do Sistema Único de Saúde (SUS) por meio dessas práticas permitiu criar um elo de confiança entre os usuários e os profissionais, mesmo diante da atuação enquanto acadêmicos. Ouvir suas dúvidas e saná-las é gratificante, pois torna a atividade interessante e atrativa, permitindo a participação das puérperas.

No decorrer do estágio, prestou-se assistência de enfermagem tanto para mães de cesariana quanto para mães de parto normal. Nesse período foi possível perceber o desconhecimento das genitoras primíparas ao lidar com seu RN no instante da amamentação. Assim, observamos a ausência de conhecimentos quanto às pegas e as posições corretas durante a amamentação, sucedido do valor nutricional do leite materno e o uso de chupetas e mamadeiras com chás e água. Frente a isso, notou-se a necessidade de intervir diante da realidade encontrada. Para tanto, foram desenvolvidas estratégias de educações em saúde voltadas para amamentação e cuidados em geral com os RNs.

As práticas alimentares infantis são feitas conforme preconizadas pela OMS, sendo levado em consideração a faixa etária das crianças. Dessa forma, os critérios das práticas alimentares estar em solicitar, permitir e não permitir os alimentos. Segundo a OMS (2008) os critérios das práticas alimentares apresentam os modos de alimentação infantis que são: AME, AMP, alimentação complementar, amamentação ao seio, alimentação por meio de fórmulas infantis.

As puérperas relataram que as ações em saúde voltadas para o AM são essenciais para adquirir conhecimento acerca da amamentação, leite materno e as orientações quanto ao uso de objetos que intervêm na amamentação adequada da criança. Assim como, a quebra de tabus e crenças por populações tradicionais, em especial a população da zona rural.

O profissional de enfermagem que atua no AC, além de atuar na assistência, também tem como uma de suas atribuições atuar com ações voltadas para orientação e ações em saúde. Esse profissional tem conhecimento e influência crucial nas vivências adquiridas durante amamentação vivida pelas mães e seus filhos, podendo reconhecer e corrigir problemas relacionados às lesões na mama, assim colaborando para o alívio de dores e desconfortos, ampliando o sucesso do 
AM (Cirico et al., 2016).

Neste contexto, Cirino et al. (2017) afirmam que a educação em saúde atua como um instrumento inseparável no decorrer desse processo, por meio de orientações indispensáveis, da ajuda ao RN, de ensinamentos acerca da amamentação, demostrando a importância do AM. Além do mais, a troca de conhecimento entre profissional e paciente através da educação em saúde é fundamental para o aprendizado das puérperas para com seus RNs.

De acordo com França et al. (2016) a educação em saúde é um dos principais meios de promoção para saúde, estando diretamente ligada com a assistência, o que nos leva ao duplo trabalho desempenhado pelos profissionais da saúde, tornando-se também educadores. Além disso, a união do conhecimento com a qualidade de vida das gestantes e seus filhos, reflete em um ciclo saudável, tornando-a transmissora dos saberes adquiridos para as futuras gerações.

Para Costa e Brito (2016) é fundamental que nos primeiros dias de vida mãe e filho fiquem juntos de forma integral, no AC, onde são passadas instruções relacionadas ao seu autocuidado e cuidados ao RN pela equipe de profissionais. A enfermagem neste setor presta assistência e instrui práticas fundamentais para a mãe e seu RN, como higiene corporal, cuidados com o coto umbilical entre outros cuidados puerperais.

Minosso et al. (2020) ressaltam a relevância do sistema de saúde em estabelecer mecanismos para promoção, contribuição e estímulo ao AM. As equipes multiprofissionais da saúde e, de modo específico a enfermagem, exercem uma atribuição fundamental na amamentação, pois além de contribuir com a assistência no AC, também ajuda no incentivo e melhorias do AM na atenção básica, desde o pré-natal até o pós-parto auxiliando na informação da introdução de alimentação suplementares no tempo correto.

Segundo Lustosa e Lima (2020) o oficio da enfermagem é assegurar mediante da promoção, acolhimento e precaução à prática do AME, não só através do conhecimento, mas também por meio da implementação de atividade que reúna e estimule as gestantes e seus familiares no decorrer do pré-natal, parto e pós-parto, colaborando para o manejo correto do AM. Além disso, é indispensável o conhecimento do enfermeiro para assegurar o vínculo materno-afetivo que assegure um bom crescimento da criança e garanta a promoção da saúde mãe/filho.

De acordo com Alves et al. (2019) apesar das medidas educativas serem essenciais durante a gestação e após é necessário bem mais, como avaliar a qualidade da sucção do RN, a pega no momento da amamentação, avaliar o tipo de mamilo se é protuso, semiprotuso ou invertido, sendo esses pontos importantes para que se tenha uma boa amamentação, proporcionando, assim, a criação e fortalecimento do vínculo mãe/filho, além de permiti-los desfrutar dos benefícios do AM.

A prática de amamentar é considerada única e essencial nos primeiros seis meses de vida para o RN, fornecendo benefícios nutricionais, imunológicos e cognitivos. Além do mais, a amamentação é algo biológico natural, mas comumente é alvo de influências de vários fatores, necessitando reafirmar o comprometimento da equipe multiprofissional da saúde no estímulo e ajuda ao AM. Diante disso, os profissionais enfermeiros(as) sempre estarão atentos no pré-natal quanto no puerpério com o intuito de prevenir possíveis intercorrências (Gomes et al., 2018).

Neste contexto, Vicente (2018) afirma que a assistência de enfermagem empregada de acordo com um modelo de enfermeiro(a) da família, permite o acompanhamento dos familiares durante todo o ciclo vital. Além do mais, o profissional de enfermagem é o mais próximo da mulher gestante, e sendo portador de informações técnicas e científicas, proporcionando ações educativas em saúde e na assistência principalmente para as grávidas e familiares. É indispensável ensinar e qualificas as mães quanto os cuidados com o posicionamento e da pega correta da criança na mamada, deixando as genitoras seguras e satisfeita ao amamentar.

Diante do exposto, percebe-se a importância do profissional enfermeiro(a) no AC, onde as gestantes devem receber todas as orientações relacionado aos benefícios do AM. Ressaltando que tais recomendações também são repassadas na atenção primária ao longo do pré-natal pela equipe de Estratégia Saúde da Família (ESF), apesar disso foi visível o 
desconhecimento sobre os benefícios e tais práticas para o sucesso do AME.

\section{Considerações Finais}

Portanto, o estágio é um período indispensável durante os últimos semestres do curso de graduação em enfermagem, garantindo ao discente atuar sob a ótica de um profissional de enfermagem, compactuando com trabalho da equipe de enfermagem que é essencial para a promoção da saúde, seja em instituições hospitalares públicas ou privadas.

A prática de atividades educativas abordando a temática do AM, através do ECS, oportunizou uma experiência única de aprimoramento profissional e pessoal, rica em informações e compartilhamento de experiências, proporcionando aos acadêmicos, por meio das orientações e trocas de conhecimento com as mães e familiares, gerar um elo de confiança com esse público.

Além disso, o estágio no AC permitiu que os acadêmicos desfrutassem de uma prática multiprofissional, por meio da interação com médicos, enfermeiros e técnicos de enfermagem, enquanto prestadores da assistência e cuidados diretos a mães e filhos. Também, foi possível presenciar na prática a realidade da equipe de enfermagem, possibilitando aos alunos desenvolver seu senso crítico.

As ações em saúde desenvolvidas no AC foram essenciais para as puérperas, através das mesmas levamos informações e conhecimentos sobre os benefícios do AM, assim como a pega correta da mama. Os materiais utilizados durante as atividades foram fixados nas paredes dos ACs e ao uso da equipe de enfermagem para futuras puérperas. Portanto, as experiências vivenciadas no AC contribuíram significativamente para o desenvolvimento pessoal e profissional dos estudantes.

Espera-se que os resultados encontrados neste trabalho permitam avanços para a continuação do AM, colaborando na preservação da mortalidade infantil e promovendo o vínculo entre mãe e RN, proporcionado pela amamentação. Os entraves do estudo foi o curto período de tempo no setor ou AC. Estudos deste tipo são essenciais, pois trazem a realidade encontrada pelos discentes nos hospitais dos interiores, além da falta de informação apresentadas pelas mães acerca da amamentação.

\section{Referências}

Alves, Y. V., Santos, J. C. J., Barreto, I. D. C., Fujinaga, C. I., \& Medeiros, A. M. C. (2019). Avaliação da sucção não nutritiva de recém-nascidos a termo e sua relação com o desempenho da mamada. Revista Brasileira de Saúde Materno Infantil, 19(3), 621-630. https://doi.org/10.1590/1806-93042019000300008

Cirico, M. O. V., Shimoda, G. T., \& Oliveira, R. N. G. D. (2016). Qualidade assistencial em aleitamento materno: implantação do indicador de trauma mamilar. Revista Gaúcha de Enfermagem, 37(4), e60546. https://doi.org/10.1590/1983-1447.2016.04.60546

Cirino, I. P., Soares, C. C., Sousa, F. V. O., Sousa, R. R. C., Lima, L. H. O., \& Oliveira, E. A. R. (2017). Educação em saúde: promovendo o aleitamento materno, um relato de experiência. Revista Interdisciplinar, 9(4), 181-186. https://revistainterdisciplinar.uninovafapi.edu. br/index.php/revinter/article/view/1077

Coca, K. P., Pinto, V. L., Westphal, F., Mania, P. N. A., \& Abrão, A. C. F. D. V. (2018). Conjunto de medidas para o incentivo do aleitamento materno exclusivo intra-hospitalar: evidências de revisões sistemáticas. Revista Paulista de Pediatria, 36(2), 214-220. https://doi.org/10.1590/1984$0462 / ; 2018 ; 36 ; 2 ; 00002$

Costa, P. B., Chagas, A. C. M. A., Joventino, E. S., Dodt, R. C. M., Oriá, M. O. B., \& Ximenes, L. B. (2013). Construção e validação de manual educativo para a promoção do aleitamento materno. Revista da Rede de Enfermagem do Nordeste, $14(6)$ 1160-1167. https://www.redalyc.org/pdf/3240/324029419012.pdf

Costa, P. F., \& Brito, R. S. (2016). Orientações ofertadas às puérperas no alojamento conjunto: revisão integrativa da literatura. Espaço para Saúde, 17(2), 237-245. https://doi.org/10.22421/15177130-2016v17n2p237

França, A. F. S., Maximin, D. A. F. M., Souto, C. G. V., \& Virgínio, N. A. (2016). Contribuições da educação em saúde por enfermeiros na adesão ao aleitamento materno. Revista de Ciências da Saúde Nova Esperança, 14(1), 73-80. https://doi.org/10.17695/revcsnevol14n1p73-80

Gomes, D. B. M., Santos, C. M., \& Rios, R. L. (2018). Amamentação e suas prerrogativas para a saúde do binômio mãe-filho. Biológicas \& Saúde, 8(27). https://doi.org/10.25242/886882720181419

Lamounier, J. A., Chaves, R. G., Rego, M. A. S., \& Bouzada, M. C. F. (2019). Iniciativa Hospital Amigo da Criança: 25 anos de experiência no Brasil. Revista Paulista de Pediatria, 37(4), 486-493. .https://dx.doi.org/10.1590/1984-0462/;2019;37;4;00004 
Lima, A. P. C., Nascimento, D. S., \& Martins, M. M. F. (2018). A prática do aleitamento materno e os fatores que levam ao desmame precoce: uma revisão integrativa. Journal of Health \& Biological Sciences, 6(2), 189-196. http://dx.doi.org/10.12662/2317-3076jhbs.v6i2.1633.p189-196.2018

Lustosa, E., \& Lima, R. N. (2020). Importância da enfermagem frente à assistência primária ao aleitamento materno exclusivo na atenção básica. Revista Brasileira Interdisciplinar de Saúde, 2(2), 93-7. https://revistarebis.rebis.com.br/index.php/rebis/article/download/96/89

Maeda, T. C., Parreira, B. D. M., Silva, S. R., \& Oliveira, A. C. D. A. (2014). Importância atribuída por puérperas às atividades desenvolvidas no prénatal. Revista de Enfermagem e Atenção à Saúde, 3(2), 6-18. http://seer.uftm.edu.br/revistaeletronica/index.php/enfer/article/view/1016

Ministério da Saúde. (2006). Lei ${ }^{\circ}$ 11.265, de 3 de janeiro de 2006: Regulamenta a comercialização de alimentos para lactentes e crianças de primeira infância e também a de produtos de puericultura correlatos. Diário Oficial da República Federativa do Brasil. http://www.planalto.gov.br/ccivil_03/_ato20042006/2006/lei/111265.htm

Ministério da Saúde. (2014). Portaria n 1.153, de 22 de maio de 2014. Redefine os critérios de habilitação da Iniciativa Hospital Amigo da Criança (IHAC), como estratégia de promoção, proteção e apoio ao aleitamento materno e à saúde integral da criança e da mulher, no âmbito do Sistema Único de Saúde (SUS). Diário Oficial da República Federativa do Brasil. http://bvsms.saude.gov.br/bvs/saudelegis/gm/2014/prt1153_22_05_2014.html

Ministério da Saúde. (2015). Saúde da criança: aleitamento materno e alimentação complementar. Secretaria de Atenção à Saúde, Departamento de Atenção Básica. https://bvsms.saude.gov.br/bvs/publicacoes/saude_crianca_aleitamento_materno_cab23.pdf

Ministério da Saúde. (2016). Portaria no 2.068, de 21 de outubro de 2016. Institui diretrizes para a organização da atenção integral e humanizada à mulher e ao recém-nascido no Alojamento Conjunto. Diário Oficial da República Federativa do Brasil. https://bvsms.saude.gov.br/bvs/ saudelegis/gm/2016/prt2068_21_10_2016.html

Minosso, K. C., Toso, B. R. G. D. O., Piva, E. K., \& Christoffel, M. M. (2020). Validação para o português da escala de conhecimento acerca do aleitamento materno. Acta Paulista de Enfermagem, 33, 1-11. https://dx.doi.org/10.37689/acta-ape/2020ao0067

Organização Mundial da Saúde. (2008). Indicadores para avaliação das práticas de alimentação de bebês e crianças pequenas: parte 1: definições: conclusões de uma reunião de consenso realizada de 6 a 8 de novembro de 2007 em Washington DC, EUA. Organização Mundial da Saúde. https://apps.who.int/iris/bitstream/handle/10665/43895/9789242596663_fre.pdf

Pasqual, K. K., Braccialli, L. A. D., \& Volponi, M. (2010). Alojamento conjunto: espaço concreto de possibilidades e o papel da equipe multiprofissional. Cogitare Enfermagem, 15(2), 334-339. https://www.redalyc.org/pdf/4836/483648971003.pdf

Rocha, E. M. A., et al., (2020). Aleitamento materno, amamentação tranquila e prazerosa: um relato de experiência. Research, Society and Development, 9(7),1-8, e155974006. https://doi.org/10.33448/rsd-v9i7.4006

Saldan, P. C., Venancio, S. I., Saldiva, S. R. D. M., Pina, J. C., \& Mello, D. F. D. (2015). Práticas de aleitamento materno de crianças menores de dois anos de idade com base em indicadores da Organização Mundial da Saúde. Revista de Nutrição, 28 (4), 409-420. https://dx.doi.org/10.1590/1415-52732015000400007

Silva, N. A. D., Avelino, M. B. M., Almeida, M. V. D., Mota, P. M. A., Sena, C. D. S., Moraes, Â. C. D., \& Oliveira, J. C. D. (2021). Os benefícios do alojamento conjunto na ótica do enfermeiro: revisão integrativa. Brazilian Journal of Development, 7(2), 15982-15991. https://doi.org/10.34117/bjdv7n2-292

Souza, C. A., Oliveira, L. F., \& Peruzzo, S. A. F. (2018). Conhecimento das puérperas sobre os benefícios da amamentação em ambiente hospitalar. Cadernos da Escola de Saúde, 18(1), 1-22 https://portaldeperiodicos.unibrasil.com.br/index.php/cadernossaude/article/view/3784

Vicente, C. M. I. (2018). Atitudes dos enfermeiros na promoção e proteção do aleitamento materno (Tese de doutorado, Instituto Politécnico de Viseu, Escola Superior de Saúde de Viseu). https://repositorio.ipv.pt/bitstream/10400.19/5059/1/Cristina_Margarida_Iglesias_Vicente_DM.pdf 